\title{
Gene expression in the adrenal glands of three spontaneously hypertensive rat substrains
}

\author{
MOHAMMAD SAID ASHENAGAR ${ }^{1}$, MASAKI TABUCHI $^{1}$, KOSHO KINOSHITA ${ }^{1}$, KANA OOSHIMA ${ }^{1}$, \\ ATSUKO NIWA $^{1}$, YUKO WATANABE ${ }^{2,3}$, MOMOKO YOSHIDA $^{2,3}$, KAZUNORI SHIMADA ${ }^{2}$, \\ TERUO YASUNAGA $^{3}$, HIROMICHI YAMANISHI ${ }^{2}$ and HIDEAKI HIGASHINO ${ }^{1}$ \\ ${ }^{1}$ Department of Pharmacology, Kinki University School of Medicine, Osaka 589-8511; \\ ${ }^{2}$ Hirakata Ryoikuen, Osaka 573-0122; ${ }^{3}$ Department of Genome Informatics, Genome Information Research Center, \\ Research Institute for Microbial Diseases, Osaka University, Osaka 565-0871, Japan
}

Received September 30, 2009; Accepted November 25, 2009

DOI: $10.3892 / \mathrm{mmr} 00000242$

\begin{abstract}
We examined gene expression profiles in rat adrenal glands using genome-wide microarray technology. Gene expression levels were determined in four rat strains, including one normotensive strain [Wistar-Kyoto (WKY)] and three substrains derived from WKY rats: spontaneously hypertensive rats (SHR), stroke-prone SHR (SHRSP) and malignant SHRSP (M-SHRSP). This study represents the first attempt at using microarrays to compare gene expression profiles in SHR, SHRSP and M-SHRSP adrenal glands, employing WKY as controls. Expression measurements were made in these four rat strains at 6 and 9 weeks of age; 6 weeks of age covers the pre-hypertensive period in SHR and SHRSP, and 9 weeks of age is the period of rapidly rising blood pressure (BP). Since the aim of this study was to identify candidate genes involved in the genesis of hypertension in the SHR substrains, we identified genes that were consistently different in their expression, isolating 87 up-regulated genes showing a more than 4-fold increase and 128 down-regulated genes showing a less than 1/4-fold decrease in at least two different experiments. We classified all these up- or down-regulated genes by their expression profiles, and searched for candidate genes.
\end{abstract}

Correspondence to: Dr Hideaki Higashino, Department of Pharmacology, Kinki University School of Medicine, Ohno-Higashi, Osaka-Sayama, Osaka 589-8511, Japan

E-mail: higasino@med.kindai.ac.jp

Abbreviations: BP, blood pressure; NA, non-annotated clones; NFA, non-functionally annotated clones; RefSeq, reference mRNA sequence; SBP, systolic blood pressure; SHR, spontaneously hypertensive rats; SHRSP, stroke-prone SHR; M-SHRSP, malignant SHRSP; WKY, normotensive Wistar-Kyoto rat, a wild-type control strain

Key words: adrenal gland, gene expression, hypertension, spontaneously hypertensive rats, stroke-prone spontaneously hypertensive rats, malignant spontaneously hypertensive rats
At 6 weeks of age, several BP-regulating genes including sparc/osteonectin (Spock2), kynureninase (Kynu), regulator of G-protein signaling $2(\operatorname{Rgs} 2)$ and gap junction protein $\alpha 1$ (Gjal) were identified as up-regulated, and urotensin 2 (Uts2), cytoplasmic epoxide hydrolase 2 (Ephx2), apelin (Apln), insulin-like growth factor 1 receptor (Igflr) and angiotensin II receptor-associated protein (Agtrap) were identified as downregulated. The Kynu and Ephx2 genes have previously been reported by other groups to be responsible for hypertension in SHR; however, our present approach identified at least seven new candidate genes.

\section{Introduction}

The polygenic nature of human essential hypertension has made it difficult to isolate the genes involved in the genesis of the disease. Microarrays are a potentially powerful tool for studying the genetics of hypertension, as they facilitate the simultaneous measurement of the expression of thousands of genes. Since inbred homozygous rodent models of human essential hypertension are ideal for microarray research, animal models of essential hypertension have recently been studied using microarrays (1).

In this study, we present a comparison of adrenal gland gene expression in three strains of hypertensive rats: spontaneously hypertensive rats (SHR) as well as two substrains derived from SHR, stroke-prone SHR (SHRSP) and malignant SHRSP (M-SHRSP) (2-4). SHR, the current paradigm for essential hypertension research, were developed in a breeding program with selection based solely on elevated blood pressure (BP) in Wistar rats (2). Normotensive descendants of Wistar rats [Wistar-Kyoto rats (WKY)], from which the SHR strain was derived, were used as controls $(2,3)$. SHRSP were established from SHR by selective inbreeding for stroke-proneness (3), and M-SHRSP were established through the brother-sister mating of selected SHRSP showing higher BP at an early stage of development for 20 generations (4). An inbred strain of M-SHRSP exhibited BP as high as $250 \mathrm{mmHg}$ or more before 14 weeks of age, and, compared to SHRSP, experienced more rapid and severe increases in BP (4). 
Table I. Blood pressure and gene expression in SHR, SHRSP and M-SHRSP.

\begin{tabular}{lccc}
\hline Rats & $\begin{array}{c}\text { Blood pressure } \\
(\mathrm{mmHg})\end{array}$ & $\begin{array}{c}>4 \text {-fold } \\
\text { up-regulated probes }\end{array}$ & $\begin{array}{r}<1 / 4 \text {-fold } \\
\text { down-regulated probes }\end{array}$ \\
\hline Six weeks of age & $130 \pm 6$ & & 4,407 \\
WKY & $136 \pm 5$ & 59 & 149 \\
SHR & $132 \pm 3$ & 112 & 74 \\
SHRSP & $160 \pm 4^{\mathrm{a}}$ & & 42 \\
M-SHRSP & & & 256 \\
Nine weeks of age & $137 \pm 5$ & 71 & 19 \\
WKY & $158 \pm 7$ & 146 & 4,947 \\
SHR & $180 \pm 10^{\mathrm{a}, \mathrm{b}}$ & 476 & \\
SHRSP & $217 \pm 10^{\mathrm{a}, \mathrm{b}}$ & & \\
M-SHRSP & & 476 & \\
Total up- and down-regulated probes & & & \\
\hline
\end{tabular}

Significant differences at $\mathrm{P}<0.001$ vs. ${ }^{a}$ WKY of the same age, and vs. bthe same subgroup of rats aged 6 weeks.

Adrenal gland secretory products, both medullary and cortical, are logical candidates for the study of hypertension, since they can directly influence cardiovascular, endocrine and sympathetic functions (5). This study represents the first attempt at using microarrays to compare gene expression profiles in SHR, SHRSP and M-SHRSP adrenal glands, employing WKY as controls. The aim of the study was to identify candidate genes involved in the genesis of hypertension in the SHR substrains.

\section{Materials and methods}

Animals and measurements. Experiments were performed using rats aged 6 and 9 weeks. WKY/Izm were employed as a wild-type control strain, and SHR/Kpo, SHRSP/Kpo and M-SHRSP/Kpo as hypertension model rats (2-4). The WKY/ Izm strain was purchased from SLC Co. (Shizuoka, Japan), and the other three substrains from the Kinki University Animal Center. All animals used in the experiment were handled with due care according to the guidelines established by the Japanese Association for Laboratory Animal Science, which comply with international rules and policies. This study was performed with the approval of the Animal Care and Use Committee of Kinki University (KAME-19-078, April 1, 2007). All possible measures were taken to minimize the pain and discomfort of the experimental animals.

Systolic blood pressure (SBP) measurements were performed using the tail-cuff method with a UR-5000 instrument (Ueda, Tokyo, Japan). Briefly, three consecutive $\mathrm{BP}$ readings were taken, and $\mathrm{BP}$ values were expressed as the mean \pm SEM. Comparisons between the means of groups were made using one-way analysis of variance (ANOVA) and Scheffe's multiple comparisons test, with differences considered significant at $\mathrm{P}<0.05$.

Tissue processing and RNA isolation. After the adrenal glands were harvested, the organs were homogenized at a pitch speed of 22 strokes/sec for $2 \mathrm{~min}$ ( 2 times) in a 2-ml plastic tube with a 5-mm-diameter glass bead using the Qiagen Tissue Lyser (Retsch Gmbh \& Co., Haan, Germany). Total RNA was extracted using an RNeasy Mini Kit (Qiagen Sciences, Germantown, MD, USA) according to the manufacturer's protocol. Prior to its use in the microarray experiments, RNA quality was verified with RNA Nano Chips (Agilent Technologies, Waldbornn, Germany) using an Agilent 2100 Bioanalyzer. Three rats each from all hypertensive and normotensive strains were used in each experiment.

Analysis of gene expression profiling using oligonucleotide arrays. To examine gene expression profiles in the adrenal glands of rats, cDNA was synthesized from $1 \mu \mathrm{g}$ of the DNase I-treated total RNA using a Low RNA Input Amplification kit (Agilent Technologies), and was subjected to hybridization through incubation using a Whole Rat Genome Microarray kit $(4 \mathrm{x} 44 \mathrm{~K})$ (Agilent Technologies) with a rotor oven (Sure-print Technology, USA) for $17 \mathrm{~h}$ at $65^{\circ} \mathrm{C}$, followed by a washing microarray. The hybridized slides were scanned with the Agilent GenPix Scanner 4000 (Agilent Technologies). Data were extracted, and the overall raw signal intensities on each array were normalized to the median value of all rat probes using BRB-Array Tool software ver. 3.7.0. (Biometric Research Branch) (6). A significance level $(\mathrm{P}<0.05)$ for each probe set was calculated using the Student's t-univariate test.

Annotation of differentially expressed genes. A BLASTN search was preformed using the NCBI RefSeq database employing the corresponding 60-nucleotide-long probes (NCBI, GEO accession, GPL7294) in order to identify homologous genes with functional annotations (7). After running the BLASTN search, clones showing a score $>50$ or an E-value $<5 \mathrm{e}-05$ were defined as annotated clones, while the remaining clones were defined as non-annotated. Some of the annotated clones, either encoding hypothetical proteins or corresponding to uncharacterized cDNA clones, were defined as non-functionally annotated. Annotated gene and protein symbols were written in italics and plain text, respectively. 


\section{Results}

Blood pressure measurements. SBP in WKY and the three SHR substrains was measured at 6 and 9 weeks of age (Table I). Although SBP was significantly higher in M-SHRSP at 6 weeks of age than in WKY, SHR and SHRSP, no significant difference was noted in SBP among WKY, SHR and SHRSP at this age (Table I). However, at 9 weeks of age, SBP in the SHR substrains gradually increased in the order of WKY $<$ SHR $<$ SHRSP $<$ M-SHRSP (Table I).

Microarray statistical results. Since the aim of this study was to identify candidate genes involved in the genesis of hypertension in SHR substrains, gene expression profiles were compared using chips containing 41,012 probes and mRNAs extracted from the adrenal glands of SHR substrains at 6 and 9 weeks of age. The number of $>4$-fold up-regulated probes was found to be increased from 4 in SHR to 59 in SHRSP and to 112 in M-SHRSP at 6 weeks of age (Table I). By contrast, the number of $<1 / 4$-fold down-regulated probes was as high as 4,407 in SHR, and was markedly decreased to 149 in SHRSP and to 74 in M-SHRSP at 6 weeks of age (Table I).

Expression levels of each probe were estimated at 6 and 9 weeks of age, meaning each probe generated at least six different bits of data (Table II). The number of up- or downregulated genes was less than the number of probes, due to redundancy in the probe sets (i.e., in some cases, two or three probes represent one gene). Genes exhibiting $>4$-fold or $<1 / 4$-fold expression in SHR substrains in comparison to WKY in at least two out of the six independent experiments were defined as up- and down-regulated genes, respectively. The 476 up-regulated probes identified 87 up-regulated genes, and the 4,947 down-regulated probes identified 128 downregulated genes (Tables I and II). As it has been reported that only a few major loci play a role in the pathogenesis of hypertension in SHR (at least three) $(8,9)$, we presumed that these $87+128$ genes embody most of the candidate genes involved in the genesis of hypertension in SHR substrains, and expected the expression of candidate genes to be up- or down-regulated well before the elevation of BP, i.e., by 6 weeks of age in SHR and/or SHRSP (Table I). Accordingly, we examined the expression profiles of the 87 up-regulated and 128 downregulated genes, and classified these genes by their expression profiles (Table II).

Classification of the 87 up-regulated genes. The 87 up-regulated genes showed 24 expression profiles, and were classified into 5 groups (Table IIA, U1 to U5). The U1 group included 4 genes expressed in SHR at 6 weeks of age, and the U2 group, 24 genes expressed in SHRSP at 6 weeks of age. The U3 group included 33 genes expressed in M-SHRSP at 6 weeks of age, the U4 group, 10 genes expressed in SHR at 9 weeks of age, and the U5 group, 16 genes expressed in SHRSP at 9 weeks of age (Table IIA).

Taking expression profiles and levels of up-regulation into consideration, each one of the 87 up-regulated genes was classified together with the results of their annotations, summarized in Table III. We focused on the 28 genes of the $\mathrm{U} 1$ and U2 groups, since these were up-regulated in SHR and/
Table II. Gene expression profiles in SHR, SHRSP and M-SHRSP.

A, 87 up-regulated genes showing 24 expression profiles.

\begin{tabular}{lrrrrrr}
\hline Group & $6 \mathrm{H}$ & $6 \mathrm{~S}$ & $6 \mathrm{M}$ & $9 \mathrm{H}$ & $9 \mathrm{~S}$ & $9 \mathrm{M}$ \\
\hline U1 (4) & 1 & 1 & 1 & 1 & 1 & 1 \\
& 1 & 1 & 1 & 0 & 0 & 0 \\
& 1 & 0 & 1 & 1 & 1 & 1 \\
U2 (24) & 1 & 0 & 1 & 0 & 0 & 0 \\
& 0 & 3 & 3 & 3 & 3 & 3 \\
& 0 & 2 & 2 & 2 & 2 & 0 \\
& 0 & 3 & 3 & 3 & 0 & 3 \\
& 0 & 1 & 1 & 0 & 1 & 0 \\
& 0 & 1 & 1 & 0 & 0 & 1 \\
& 0 & 11 & 11 & 0 & 0 & 0 \\
& 0 & 1 & 0 & 1 & 1 & 1 \\
& 0 & 1 & 0 & 1 & 0 & 1 \\
U3 (33) & 0 & 1 & 0 & 1 & 0 & 0 \\
& 0 & 0 & 6 & 6 & 6 & 6 \\
& 0 & 0 & 4 & 4 & 4 & 0 \\
& 0 & 0 & 3 & 3 & 0 & 3 \\
& 0 & 0 & 4 & 4 & 0 & 0 \\
& 0 & 0 & 4 & 0 & 4 & 4 \\
& 0 & 0 & 2 & 0 & 2 & 0 \\
U5 (10) & 0 & 0 & 10 & 0 & 0 & 10 \\
& 0 & 0 & 0 & 4 & 4 & 4 \\
& 0 & 0 & 0 & 2 & 2 & 0 \\
& 0 & 0 & 0 & 0 & 16 & 16 \\
& 0 & 0 & 4 & 0 & 4 \\
& 0 & & & & &
\end{tabular}

B, 128 down-regulated genes showing 18 expression profiles.

\begin{tabular}{lrrrrrr}
\hline Group & $6 \mathrm{H}$ & $6 \mathrm{~S}$ & $6 \mathrm{M}$ & $9 \mathrm{H}$ & $9 \mathrm{~S}$ & $9 \mathrm{M}$ \\
\hline D1 (98) & 2 & 2 & 2 & 2 & 2 & 2 \\
& 1 & 1 & 1 & 0 & 0 & 1 \\
& 24 & 24 & 24 & 0 & 0 & 0 \\
& 52 & 52 & 0 & 0 & 0 & 0 \\
& 1 & 0 & 1 & 1 & 1 & 1 \\
& 1 & 0 & 1 & 0 & 1 & 0 \\
& 15 & 0 & 15 & 0 & 0 & 0 \\
& 1 & 0 & 0 & 1 & 0 & 1 \\
D2 (9) & 1 & 0 & 0 & 0 & 1 & 0 \\
& 0 & 1 & 1 & 1 & 1 & 1 \\
& 0 & 1 & 1 & 0 & 0 & 1 \\
& 0 & 6 & 6 & 0 & 0 & 0 \\
D3 (1) & 0 & 1 & 0 & 1 & 1 & 0 \\
D4 (18) & 0 & 0 & 1 & 1 & 1 & 0 \\
& 0 & 0 & 0 & 7 & 7 & 7 \\
& 0 & 0 & 0 & 10 & 10 & 0 \\
D5 (2) & 0 & 0 & 0 & 1 & 0 & 1 \\
\hline
\end{tabular}

Up- or down-regulated genes were classified into 5 groups according to expression profile (U1-U5 and D1-D5). Numbers in parentheses indicate the number of genes belonging to each group. 6, 6 weeks of age; 9, 9 weeks of age; H, SHR; S, SHRSP; M, M-SHRSP. Values from 0 to 52 indicate the number of up- or down-regulated genes. 
Table III. The 87 genes up-regulated in SHR, SHRSP and M-SHRSP.

\begin{tabular}{|c|c|c|c|c|c|c|c|c|c|}
\hline $\mathrm{Gr}$ & Probe ID & $6 \mathrm{H}$ & $6 \mathrm{~S}$ & $6 \mathrm{M}$ & $9 \mathrm{H}$ & $9 \mathrm{~S}$ & $9 \mathrm{M}$ & Fold-change & Gene or protein name (symbol) \\
\hline U1 & A_44_P152177 & 1 & 1 & 1 & 1 & 1 & 1 & $23.2-4.9$ & *1, Sparc/osteonectin (Spock2) \\
\hline U1 & A_44_P863709 & 1 & 1 & 1 & 0 & 0 & 0 & $27.3-7.0$ & NA (40), hypothetical protein \\
\hline $\mathrm{U} 1$ & A_44_P245616 & 1 & 0 & 1 & 1 & 1 & 1 & $47.6-9.8$ & Nidogen 1 (Nid1) \\
\hline $\mathrm{U} 1$ & A_44_P203564 & 1 & 0 & 1 & 0 & 0 & 0 & 9.2 and 6.1 & Otospiralin (Otos) \\
\hline $\mathrm{U} 2$ & A_44_P560710 & 0 & 1 & 1 & 1 & 1 & 1 & $37.1-8.7$ & NA (38), stem cell tumor (CpipJ_CPIJ003969) \\
\hline $\mathrm{U} 2$ & A_42_P826202 & 0 & 1 & 1 & 1 & 1 & 1 & $12.0-4.2$ & Zinc finger protein 597 (Znf597) \\
\hline $\mathrm{U} 2$ & A_42_P703548 & 0 & 1 & 1 & 1 & 1 & 1 & $11.9-6.5$ & ${ }^{*} 2$, Kynureninase (Kynu) \\
\hline $\mathrm{U} 2$ & A_44_P578428 & 0 & 1 & 1 & 1 & 1 & 0 & $11.7-4.5$ & NA (38), CNE04290 \\
\hline $\mathrm{U} 2$ & A_44_P640472 & 0 & 1 & 1 & 1 & 1 & 0 & $4.9-4.1$ & NA (38), ribosomal protein \\
\hline $\mathrm{U} 2$ & A_44_P495480 & 0 & 1 & 1 & 1 & 0 & 1 & $33.2-7.8$ & FBJ osteosarcoma oncogene homolog (c-Fos) \\
\hline $\mathrm{U} 2$ & A_43_P11932 & 0 & 1 & 1 & 1 & 0 & 1 & $25.7-4.1$ & Nuclear receptor subfamily $4 \mathrm{~A} 3(\mathrm{Nr} 4 \mathrm{a} 3)$ \\
\hline $\mathrm{U} 2$ & A_44_P1033521 & 0 & 1 & 1 & 1 & 0 & 1 & $17.9-6.6$ & Trichorhinophalangeal syndrome I (Trps1) \\
\hline $\mathrm{U} 2$ & A_44_P603503 & 0 & 1 & 1 & 0 & 1 & 0 & $21.7-10.2$ & Oxidoreductase NAD-binding domain containing 1 (Oxnad1) \\
\hline $\mathrm{U} 2$ & A_44_P396202 & 0 & 1 & 1 & 0 & 0 & 1 & $12.2-6.7$ & Interferon-inducible GTPase (RGD1309362) \\
\hline $\mathrm{U} 2$ & A_44_P455271 & 0 & 1 & 1 & 0 & 0 & 0 & 42.4 and 9.9 & NA (38), CCMP1335 \\
\hline $\mathrm{U} 2$ & A_44_P406636 & 0 & 1 & 1 & 0 & 0 & 0 & 17.9 and 7.7 & ATP-binding cassette, sub-family A (ABC1), 1 (Abca1) \\
\hline $\mathrm{U} 2$ & A_44_P464942 & 0 & 1 & 1 & 0 & 0 & 0 & 12.5 and 8.3 & NA (38), TVAG_318060 \\
\hline $\mathrm{U} 2$ & A_43_P14131 & 0 & 1 & 1 & 0 & 0 & 0 & 9.0 and 5.0 & ${ }^{*} 3$, Regulator of G-protein signaling 2 ( $\left.\operatorname{Rgs} 2\right)$ \\
\hline $\mathrm{U} 2$ & A_44_P472989 & 0 & 1 & 1 & 0 & 0 & 0 & 7.6 and 5.4 & Prostaglandin-endoperoxide synthase 2 (Ptgs2) \\
\hline $\mathrm{U} 2$ & A_42_P615837 & 0 & 1 & 1 & 0 & 0 & 0 & 7.3 and 4.2 & Creatine kinase, mitochondrial 2, sarcomeric (Ckmt2) \\
\hline $\mathrm{U} 2$ & A_44_P970369 & 0 & 1 & 1 & 0 & 0 & 0 & 6.4 and 5.6 & Heparan sulfate 2-O- sulfotransferase 1 (Hs2st1) \\
\hline $\mathrm{U} 2$ & A_44_P473217 & 0 & 1 & 1 & 0 & 0 & 0 & 6.1 and 5.4 & NFA, RIKEN cDNA 4930455F23 (RGD1309708) \\
\hline $\mathrm{U} 2$ & A_44_P777181 & 0 & 1 & 1 & 0 & 0 & 0 & 5.9 and 5.2 & RAB22A, member RAS oncogene family (Rab22a) \\
\hline $\mathrm{U} 2$ & A_44_P176831 & 0 & 1 & 1 & 0 & 0 & 0 & 5.6 and 4.3 & Met proto-oncogene (Met) \\
\hline $\mathrm{U} 2$ & A_44_P161052 & 0 & 1 & 1 & 0 & 0 & 0 & 4.5 and 4.3 & 3-Ketoacyl-CoA thiolase B peroxisomal precursor (RGD1562373) \\
\hline $\mathrm{U} 2$ & A_43_P16225 & 0 & 1 & 0 & 1 & 1 & 1 & $5.9-4.6$ & NA (40), myosin, heavy chain 11, smooth muscle (MYH11) \\
\hline $\mathrm{U} 2$ & A_44_P354699 & 0 & 1 & 0 & 1 & 0 & 1 & $8.5-7.3$ & CTD small phosphatase like 2, transcript variant 1 (CTDSPL2) \\
\hline $\mathrm{U} 2$ & A_44_P836591 & 0 & 1 & 0 & 1 & 0 & 0 & 7.8 and 9.6 & *4, Gap junction protein, $\alpha 1$ (Gja1) \\
\hline $\mathrm{U} 3$ & A_44_P402980 & 0 & 0 & 1 & 1 & 1 & 1 & $49.6-9.0$ & NK6 homeobox 2 (Nkx6-2) \\
\hline $\mathrm{U} 3$ & A_42_P686756 & 0 & 0 & 1 & 1 & 1 & 1 & $36.4-14.7$ & NFA, RIKEN cDNA 1110032A04 gene (1110032A04Rik) \\
\hline $\mathrm{U} 3$ & A_44_P553498 & 0 & 0 & 1 & 1 & 1 & 1 & $33.8-8.7$ & NA (48), carbonyl reductase 1 (Cbr1) \\
\hline $\mathrm{U} 3$ & A_44_P520159 & 0 & 0 & 1 & 1 & 1 & 1 & $12.9-4.8$ & Purinergic receptor P2Y, G-protein coupled 2 (P2ry2) \\
\hline $\mathrm{U} 3$ & A_44_P670594 & 0 & 0 & 1 & 1 & 1 & 1 & $10.8-4.5$ & NA (42) hypothetical protein CBG12924 partial \\
\hline $\mathrm{U} 3$ & A_42_P537051 & 0 & 0 & 1 & 1 & 1 & 1 & $5.8-4.6$ & Family with sequence similarity 70B (Fam70b) \\
\hline $\mathrm{U} 3$ & A_44_P653949 & 0 & 0 & 2 & 1 & 1 & 0 & $23.3-8.4$ & NA (40), hypothetical protein \\
\hline $\mathrm{U} 3$ & A_44_P777328 & 0 & 0 & 2 & 1 & 1 & 0 & $15.2-4.9$ & Phosphatidylinositol 3 kinase, reg sub, polypeptide 3 (Pik3r3) \\
\hline $\mathrm{U} 3$ & A_44_P840366 & 0 & 0 & 1 & 1 & 1 & 0 & $10.5-7.3$ & NA (38), LOC100072399 \\
\hline $\mathrm{U} 3$ & A_44_P702222 & 0 & 0 & 1 & 1 & 1 & 0 & $8.6-5.2$ & NFA, hypothetical protein, LOC100286928 \\
\hline $\mathrm{U} 3$ & A_42_P750683 & 0 & 0 & 2 & 1 & 0 & 1 & $22.7-10.7$ & Cysteine-rich, angiogenic inducer, 61 (Cyr61) \\
\hline $\mathrm{U} 3$ & A_43_P11531 & 0 & 0 & 1 & 1 & 0 & 1 & $15.7-4.6$ & Somatostatin receptor 1 (Sstr1) \\
\hline $\mathrm{U} 3$ & A_44_P189363 & 0 & 0 & 2 & 1 & 0 & 1 & 8.64 .5 & Endothelial cell-specific molecule 1 (Esm1) \\
\hline $\mathrm{U} 3$ & A_44_P1030258 & 0 & 0 & 1 & 1 & 0 & 0 & 6.3 and 6.3 & Cannabinoid receptor $1(\mathrm{Cnr} 1)$ \\
\hline $\mathrm{U} 3$ & A_44_P142401 & 0 & 0 & 1 & 1 & 0 & 0 & 5.8 and 4.7 & Family with sequence similarity 184B (Fam184b) \\
\hline $\mathrm{U} 3$ & A_44_P659416 & 0 & 0 & 1 & 1 & 0 & 0 & 5.0 and 4.5 & NA (36), CD36_04040 \\
\hline $\mathrm{U} 3$ & A_44_P732150 & 0 & 0 & 1 & 1 & 0 & 0 & 4.6 and 4.4 & NFA, expressed sequence C 77370 \\
\hline $\mathrm{U} 3$ & A_43_P12125 & 0 & 0 & 1 & 0 & 1 & 1 & $34.8-10.9$ & Jun B proto-oncogene (Junb) \\
\hline $\mathrm{U} 3$ & A_44_P868694 & 0 & 0 & 1 & 0 & 1 & 1 & $15.4-5.7$ & NA (36), hypothetical protein (GSPATT00018902001) \\
\hline $\mathrm{U} 3$ & A_42_P784172 & 0 & 0 & 1 & 0 & 1 & 1 & $9.6-4.3$ & Vomeromodulin (LOC690507) \\
\hline $\mathrm{U} 3$ & A_44_P475661 & 0 & 0 & 1 & 0 & 1 & 1 & $7.1-4.3$ & Integrator complex subunit 7 (Ints7) \\
\hline $\mathrm{U} 3$ & A_44_P731793 & 0 & 0 & 1 & 0 & 1 & 0 & 6.5 and 6.2 & NA (40), TVAG_113750 \\
\hline $\mathrm{U} 3$ & A_44_P668233 & 0 & 0 & 1 & 0 & 1 & 0 & 5.6 and 4.8 & NA (36), DHX9 \\
\hline $\mathrm{U} 3$ & A_44_P746102 & 0 & 0 & 1 & 0 & 0 & 1 & 13.3 and 4.8 & NA (40), pyrroline-5-carboxylate reductase partial \\
\hline $\mathrm{U} 3$ & A_42_P701060 & 0 & 0 & 1 & 0 & 0 & 1 & 8.4 and 8.3 & One cut homeobox 1 (Onecut1) \\
\hline $\mathrm{U} 3$ & A_42_P640277 & 0 & 0 & 1 & 0 & 0 & 1 & 5.7 and 4.7 & Ectodermal-neural cortex 1 (Enc1) \\
\hline $\mathrm{U} 3$ & A_44_P716208 & 0 & 0 & 1 & 0 & 0 & 1 & 5.3 and 4.2 & NA (38), GH17134 (DgrilGH17134) \\
\hline
\end{tabular}


Table III. Continued.

\begin{tabular}{|c|c|c|c|c|c|c|c|c|c|}
\hline $\mathrm{Gr}$ & Probe ID & $6 \mathrm{H}$ & $6 \mathrm{~S}$ & $6 \mathrm{M}$ & $9 \mathrm{H}$ & $9 \mathrm{~S}$ & $9 \mathrm{M}$ & Fold-change & Gene or protein name (symbol) \\
\hline $\mathrm{U} 3$ & A_44_P652899 & 0 & 0 & 1 & 0 & 0 & 1 & 5.2 and 4.6 & Glycoprotein m6a (Gpm6a) \\
\hline $\mathrm{U} 3$ & A_44_P900835 & 0 & 0 & 1 & 0 & 0 & 1 & 5.1 and 4.0 & NA (38), misc_RNA (CSMD3), partial \\
\hline $\mathrm{U} 3$ & A_44_P730958 & 0 & 0 & 1 & 0 & 0 & 1 & 5.0 and 4.9 & Eyes absent 1 homolog (Drosophila) (Eya1) \\
\hline $\mathrm{U} 3$ & A_44_P634618 & 0 & 0 & 1 & 0 & 0 & 1 & 5.0 and 4.0 & Syntaxin binding protein 6 (amisyn) (Stxbp6) \\
\hline $\mathrm{U} 3$ & A_44_P715524 & 0 & 0 & 1 & 0 & 0 & 1 & 4.5 and 4.2 & B-cell CLL/lymphoma 7A (Bcl7a) \\
\hline $\mathrm{U} 3$ & A_44_P793124 & 0 & 0 & 1 & 0 & 0 & 1 & 4.4 and 4.2 & Zinc finger and BTB domain containing 41 (Zbtb41) \\
\hline $\mathrm{U} 4$ & A_43_P22478 & 0 & 0 & 0 & 1 & 1 & 1 & $14.8-8.3$ & Dual-specificity tyr(Y)-phosphorylation regulated kinase 3 (Dyrk3) \\
\hline U4 & A_44_P534791 & 0 & 0 & 0 & 1 & 1 & 1 & $10.6-5.7$ & Methyltransferase like 2 (Mettl2) \\
\hline U4 & A_44_P240696 & 0 & 0 & 0 & 1 & 1 & 1 & 7.3-4.8 & C-type natriuretic peptide precursor (Cnp) \\
\hline $\mathrm{U} 4$ & A_42_P605711 & 0 & 0 & 0 & 1 & 1 & 1 & 4.9-4.2 & Family with sequence similarity 46A (Fam46a) \\
\hline $\mathrm{U} 4$ & A_43_P13199 & 0 & 0 & 0 & 1 & 1 & 0 & 12.9 and 11.4 & Folate receptor 1 (Folr1) \\
\hline U4 & A_42_P645923 & 0 & 0 & 0 & 1 & 1 & 0 & 5.3 and 4.2 & Cystathionine $\beta$ synthase (Cbs) \\
\hline $\mathrm{U} 4$ & A_44_P808922 & 0 & 0 & 0 & 1 & 0 & 1 & 6.4 and 4.4 & POU domain, class 4 , transcription factor 1 (Pou $4 \mathrm{f} 1)$ \\
\hline U4 & A_42_P719221 & 0 & 0 & 0 & 1 & 0 & 1 & 6.3 and 5.5 & Vasoactive intestinal peptide (Vip) \\
\hline U4 & A_44_P199028 & 0 & 0 & 0 & 1 & 0 & 1 & 6.3 and 4.5 & Dickkopf homolog 1 (Dkk1) \\
\hline $\mathrm{U} 4$ & A_42_P694679 & 0 & 0 & 0 & 1 & 0 & 1 & 6.0 and 4.7 & Histone deacetylase 4 (Hdac4) \\
\hline U5 & A_44_P556895 & 0 & 0 & 0 & 0 & 1 & 1 & 35.8 and 15.8 & DNA-damage-inducible transcript 4 (Ddit4) \\
\hline U5 & A_44_P351211 & 0 & 0 & 0 & 0 & 1 & 1 & 18.3 and 16.9 & Pleckstrin homology-like domain, family A, member 1 (Phlda1) \\
\hline U5 & A_44_P306581 & 0 & 0 & 0 & 0 & 1 & 1 & 14.2 and 13.0 & Growth differentiation factor 5 (Gdf5) \\
\hline U5 & A_42_P548410 & 0 & 0 & 0 & 0 & 1 & 1 & 11.3 and 10.9 & Acyl-CoA thioesterase $1($ Acot 1$)$ \\
\hline U5 & A_44_P268915 & 0 & 0 & 0 & 0 & 1 & 1 & 9.7 and 6.8 & Phospholipase B domain containing 1 (Plbd1) \\
\hline U5 & A_43_P18366 & 0 & 0 & 0 & 0 & 1 & 1 & 7.0 and 4.6 & Torsin family 1 , member B (Tor $1 b)$ \\
\hline U5 & A_44_P527238 & 0 & 0 & 0 & 0 & 1 & 1 & 6.7 and 4.9 & Solute carrier family 25 (Slc25a25) \\
\hline U5 & A_42_P538483 & 0 & 0 & 0 & 0 & 1 & 1 & 6.5 and 4.9 & Ring finger protein $40(\operatorname{Rnf} 40)$ \\
\hline U5 & A_44_P506662 & 0 & 0 & 0 & 0 & 1 & 1 & 6.4 and 4.3 & Transcriptional regulating factor 1 (Trerf1) \\
\hline U5 & A_43_P15888 & 0 & 0 & 0 & 0 & 1 & 1 & 6.2 and 4.5 & Angiomotin like 2 (Amotl2) \\
\hline U5 & A_44_P308369 & 0 & 0 & 0 & 0 & 1 & 1 & 6.0 and 5.0 & UDP-glucose glycoprotein glucosyltransferase 2 (Uggt2) \\
\hline U5 & A_42_P662710 & 0 & 0 & 0 & 0 & 1 & 1 & 5.6 and 4.2 & Death effector domain-containing (Dedd) \\
\hline U5 & A_44_P445575 & 0 & 0 & 0 & 0 & 1 & 1 & 5.5 and 5.5 & Iroquois related homeobox 2 (Irx2) \\
\hline U5 & A_44_P257518 & 0 & 0 & 0 & 0 & 1 & 1 & 5.4 and 4.6 & Insulin receptor substrate 2 (Irs2) \\
\hline U5 & A_44_P547236 & 0 & 0 & 0 & 0 & 1 & 1 & 5.2 and 4.1 & Tetraspanin 33 (Tspan33) \\
\hline U5 & A_44_P251124 & 0 & 0 & 0 & 0 & 1 & 1 & 5.0 and 4.3 & Protein tyrosine phosphatase, non-receptor type 18 (Ptpn18) \\
\hline
\end{tabular}

Gr, up-regulated U1, U2, U3, U4 and U5 groups; Probe ID, Agilent probe ID (NCBI, GEO accession, GPL7294). Numbers 0, 1 and 2 indicate the number of probes showing positive results; Fold-change, $>4$-fold up-regulation. NA, non-annotated. Numbers in parentheses indicate homology scores $<50$; NFA indicates annotated with homology scores $>50$, but no functional information. Other symbols or abbreviations are as described in Table II.

or SHRSP at 6 weeks of age and were expected to include the candidate genes. They comprised 20 functionally annotated and 8 non-functionally annotated genes (Table III, U1 and U2). Functionally annotated genes included genes reported to participate in BP control, such as sparc/osteonectin (Spock2), kynureninase (Kynu) and regulator of G-protein signaling 2 (Rgs2), as well as gap junction protein $\alpha 1$ (Gjal) (Table III, *1 to *4) $(10-13)$.

Classification of the 128 down-regulated genes. The 128 down-regulated genes showed 18 expression profiles and were classified into 5 groups (Table IIB, D1-D5). The D1 group included 98 genes expressed in SHR at 6 weeks of age, and the D2 group, 9 genes expressed in SHRSP at 6 weeks of age. The D3 group contained 1 gene expressed in M-SHRSP at 6 weeks of age, the D4 group, 18 genes expressed in SHR at 9 weeks of age, and the D5 group, 2 genes expressed in SHRSP at 9 weeks of age, respectively (Table IIB).

Taking expression profiles and levels of down-regulation into consideration, each one of the 128 genes was classified together with the results of their annotations, summarized in Table IV. We focused on the 107 genes of the D1 and D2 groups, since these were down-regulated in SHR and/ or SHRSP at 6 weeks of age and were expected to include the candidate genes. They comprised 77 functionally annotated and 30 non-annotated genes (Table IV, D1 and D2). Functionally annotated genes included genes reported to participate in BP control, such as urotensin 2 (Uts2), cytoplasmic epoxide hydrolase 2 (Ephx2), apelin (Apln), insulin-like growth factor 1 receptor (Igflr) and angiotensin II type I receptor-associated protein (Agtrap) (14-18) (Table IV, *1 to *5). 
Table IV. The 128 genes down-regulated in SHR, SHRSP and M-SHRSP.

\begin{tabular}{|c|c|c|c|c|c|c|c|c|c|}
\hline $\mathrm{Gr}$ & Probe ID & $6 \mathrm{H}$ & $6 \mathrm{~S}$ & $6 \mathrm{M}$ & $9 \mathrm{H}$ & $9 \mathrm{~S}$ & $9 \mathrm{M}$ & Fold-change & Gene or protein name (symbol) \\
\hline D1 & A_44_P637734 & 2 & 3 & 3 & 1 & 1 & 1 & $0.07-0.16$ & DEAD/H box polypeptide 11 (Ddx11) \\
\hline D1 & A_44_P345207 & 1 & 1 & 1 & 1 & 1 & 1 & $0.08-0.21$ & ER-Golgi intermediate compartment protein 1 (Ergic1) \\
\hline D1 & A_44_P362486 & 1 & 1 & 1 & 1 & 0 & 1 & $0.14-0.25$ & Actin-binding LIM protein 1 (Ablim1) \\
\hline D1 & A_42_P508921 & 1 & 1 & 1 & 0 & 0 & 0 & $0.01-0.02$ & Aquaporin 3 (Aqp3) \\
\hline D1 & A_44_P531908 & 1 & 1 & 1 & 0 & 0 & 0 & $0.02-0.07$ & ${ }^{*} 1$, Urotensin 2 (Uts2) \\
\hline D1 & A_43_P11634 & 2 & 2 & 2 & 0 & 0 & 0 & $0.02-0.13$ & GDNF family receptor $\alpha 1$ (Gfra1) \\
\hline D1 & A_44_P422233 & 1 & 1 & 1 & 0 & 0 & 0 & $0.03-0.06$ & NA (38), hypothetical protein, CaO19_10805 \\
\hline D1 & A_44_P562496 & 1 & 1 & 1 & 0 & 0 & 0 & $0.04-0.11$ & DEAD box polypeptide 42 protein (Ddx 42$)$ \\
\hline D1 & A_42_P803590 & 1 & 1 & 1 & 0 & 0 & 0 & 0.04-0.19 & NFA, B0432.8 (LOC289378) \\
\hline D1 & A_42_P640641 & 1 & 1 & 1 & 0 & 0 & 0 & $0.05-0.15$ & Homer homolog 2 (Homer2) \\
\hline D1 & A_44_P213133 & 1 & 1 & 1 & 0 & 0 & 0 & $0.05-0.17$ & Endonuclease G (Endog) \\
\hline D1 & A_44_P398060 & 2 & 1 & 2 & 0 & 0 & 0 & $0.05-0.18$ & Neuronal cell adhesion molecule (Nrcam) \\
\hline D1 & A_44_P577208 & 1 & 1 & 1 & 0 & 0 & 0 & $0.06-0.08$ & NA (38), zf(c2h2)-31 \\
\hline D1 & A_44_P531870 & 1 & 1 & 1 & 0 & 0 & 0 & 0.07-0.09 & *2, Epoxide hydrolase 2, cytoplasmic (Ephx2) \\
\hline D1 & A_44_P808578 & 1 & 1 & 1 & 0 & 0 & 0 & $0.07-0.10$ & NA (38), LOC100214468 \\
\hline D1 & A_42_P809869 & 1 & 1 & 1 & 0 & 0 & 0 & $0.07-0.17$ & Thiosulfate sulfurtransferase, mitochondrial (Tst) \\
\hline D1 & A_44_P147254 & 1 & 1 & 1 & 0 & 0 & 0 & $0.07-0.22$ & Cartilage intermediate layer protein 2 (Cilp2) \\
\hline D1 & A_44_P402948 & 1 & 1 & 1 & 0 & 0 & 0 & $0.08-0.15$ & NA (36), LOC717608 \\
\hline D1 & A_44_P108063 & 1 & 1 & 1 & 0 & 0 & 0 & $0.09-0.15$ & Rhomboid 5 homolog 1 (Rhbdf1) \\
\hline D1 & A_44_P823114 & 1 & 1 & 1 & 0 & 0 & 0 & $0.10-0.16$ & NA (40), Hypothetical protein \\
\hline D1 & A_44_P1022458 & 1 & 1 & 1 & 0 & 0 & 0 & $0.12-0.24$ & Tubulin, $\beta 6$ (Tubb6) \\
\hline D1 & A_42_P460340 & 2 & 1 & 1 & 0 & 0 & 0 & $0.14-0.24$ & Mal, T-cell differentiation protein 2 (Mal2) \\
\hline D1 & A_44_P610294 & 1 & 1 & 1 & 0 & 0 & 0 & $0.15-0.20$ & NA (36), BRAFLDRAFT_119740 \\
\hline D1 & A_44_P111865 & 1 & 1 & 1 & 0 & 0 & 0 & $0.15-0.22$ & Cytochrome P450, 2A, 3a (Cyp2a3a) \\
\hline D1 & A_44_P850331 & 1 & 1 & 1 & 0 & 0 & 0 & $0.17-0.23$ & NA (38), GI20648 \\
\hline D1 & A_44_P298730 & 1 & 1 & 1 & 0 & 0 & 0 & $0.17-0.24$ & NA (38), Cys-rich prot.2-bind protein \\
\hline D1 & A_43_P10482 & 1 & 1 & 1 & 0 & 0 & 0 & $0.19-0.22$ & NA (38), CBS138 \\
\hline D1 & A_44_P214900 & 1 & 1 & 0 & 0 & 0 & 0 & 0.04 and 0.15 & Pregnancy-zone protein (Pzp) \\
\hline D1 & A_42_P637189 & 1 & 1 & 0 & 0 & 0 & 0 & 0.04 and 0.16 & *3, Apelin (Apln) \\
\hline D1 & A_44_P945741 & 1 & 1 & 0 & 0 & 0 & 0 & 0.05 and 0.14 & NA (38), TVAG_290990 \\
\hline D1 & A_44_P119527 & 1 & 1 & 0 & 0 & 0 & 0 & 0.06 and 0.22 & Mediator complex subunit 17 (Med17) \\
\hline D1 & A_44_P740817 & 1 & 1 & 0 & 0 & 0 & 0 & 0.07 and 0.20 & Ubiquitin-conjugating enzyme E2Z (Ube2z) \\
\hline D1 & A_44_P387005 & 1 & 1 & 0 & 0 & 0 & 0 & 0.07 and 0.22 & 1-Aminocyclopropane-1-carboxylate synthase homolog (Accs) \\
\hline D1 & A_44_P234315 & 1 & 1 & 0 & 0 & 0 & 0 & 0.07 and 0.24 & NA (38), Misc_RNA (EMR2) \\
\hline D1 & A_44_P1039616 & 1 & 1 & 0 & 0 & 0 & 0 & 0.08 and 0.20 & Cd300D antigen (LOC498022) \\
\hline D1 & A_44_P486624 & 2 & 2 & 0 & 0 & 0 & 0 & 0.08 and 0.22 & Pleckstrin homology domain containing, A, 6 (Plekha6) \\
\hline D1 & A_44_P1041460 & 1 & 1 & 0 & 0 & 0 & 0 & 0.08 and 0.23 & Ras-related GTP binding D (Rragd) \\
\hline D1 & A_44_P181560 & 1 & 1 & 0 & 0 & 0 & 0 & 0.10 and 0.13 & Coiled-coil domain containing 95 (Ccdc95) \\
\hline D1 & A_42_P549451 & 1 & 1 & 0 & 0 & 0 & 0 & 0.10 and 0.19 & Fatty acid binding protein 6 , ileal (Fabp6) \\
\hline D1 & A_44_P507343 & 1 & 1 & 0 & 0 & 0 & 0 & 0.10 and 0.21 & NA (38), Lrp12 \\
\hline D1 & A_44_P701521 & 1 & 1 & 0 & 0 & 0 & 0 & 0.10 and 0.21 & NA (34), SORBIDRAFT_02g013130 \\
\hline D1 & A_44_P792797 & 1 & 1 & 0 & 0 & 0 & 0 & 0.11 and 0.18 & NA (38), A1CF \\
\hline D1 & A_44_P481160 & 1 & 1 & 0 & 0 & 0 & 0 & 0.11 and 0.21 & Wingless-type MMTV integration site 5A (Wnt5a) \\
\hline D1 & A_43_P18817 & 1 & 1 & 0 & 0 & 0 & 0 & 0.11 and 0.21 & Intraflagellar transport 52 homolog (LOC684302) \\
\hline D1 & A_44_P899288 & 1 & 1 & 0 & 0 & 0 & 0 & 0.11 and 0.22 & Leucine rich repeat and sterile $\alpha$ motif containing 1 (Lrsam1) \\
\hline D1 & A_42_P548791 & 1 & 1 & 0 & 0 & 0 & 0 & 0.11 and 0.23 & Translin (Tsn) \\
\hline D1 & A_44_P562830 & 1 & 1 & 0 & 0 & 0 & 0 & 0.12 and 0.17 & Dual specificity phosphatase-like 15 (Dusp15) \\
\hline D1 & A_42_P520497 & 1 & 1 & 0 & 0 & 0 & 0 & 0.13 and 0.15 & Solute carrier family 2 (facilitated glucose transporter), 5 (Slc2a5) \\
\hline D1 & A_44_P653343 & 1 & 1 & 0 & 0 & 0 & 0 & 0.13 and 0.22 & NA (40), NEMVEDRAFT_v1g176880 \\
\hline D1 & A_43_P14506 & 1 & 1 & 0 & 0 & 0 & 0 & 0.14 and 0.14 & Ankyrin repeat domain 34B (Ankrd34b) \\
\hline D1 & A_42_P548889 & 1 & 1 & 0 & 0 & 0 & 0 & 0.14 and 0.20 & Replication protein-binding trans-activator RBT1 (MGC108974) \\
\hline D1 & A_42_P627572 & 1 & 1 & 0 & 0 & 0 & 0 & 0.14 and 0.22 & Mediator of DNA damage checkpoint 1 (Mdc1) \\
\hline D1 & A_44_P1037892 & 1 & 1 & 0 & 0 & 0 & 0 & 0.15 and 0.15 & Citrin (RGD1565889) \\
\hline D1 & A_44_P274976 & 1 & 1 & 0 & 0 & 0 & 0 & 0.15 and 0.16 & NA (38), Hypothetical protein \\
\hline D1 & A_44_P871196 & 1 & 1 & 0 & 0 & 0 & 0 & 0.15 and 0.16 & NA (40), BRAFLDRAFT_277232 \\
\hline D1 & A_44_P996952 & 1 & 1 & 0 & 0 & 0 & 0 & 0.15 and 0.18 & Family with sequence similarity 113 , member B (Fam113b) \\
\hline D1 & A_42_P807866 & 1 & 1 & 0 & 0 & 0 & 0 & 0.16 and 0.16 & Reversion induced LIM gene (Ril) \\
\hline
\end{tabular}


Table IV. Continued.

\begin{tabular}{|c|c|c|c|c|c|c|c|c|c|}
\hline $\mathrm{Gr}$ & Probe ID & $6 \mathrm{H}$ & $6 \mathrm{~S}$ & $6 \mathrm{M}$ & $9 \mathrm{H}$ & $9 \mathrm{~S}$ & $9 \mathrm{M}$ & Fold-change & Gene or protein name (symbol) \\
\hline D1 & A_42_P804387 & 1 & 1 & 0 & 0 & 0 & 0 & 0.16 and 0.22 & Ly6/Plaur domain containing 3 (Lypd3) \\
\hline D1 & A_44_P730235 & 1 & 1 & 0 & 0 & 0 & 0 & 0.17 and 0.18 & Tubulin tyrosine ligase-like family 9 (Tt1l9) \\
\hline D1 & A_44_P932360 & 1 & 1 & 0 & 0 & 0 & 0 & 0.17 and 0.22 & N-acetyltransferase 13 (Nat13) \\
\hline D1 & A_43_P12888 & 1 & 1 & 0 & 0 & 0 & 0 & 0.17 and 0.25 & Platelet-activating factor acetylhydrolase $1 \mathrm{~b} 3$ (Pafah1b3) \\
\hline D1 & A_44_P925674 & 2 & 2 & 0 & 0 & 0 & 0 & 0.18 and 0.22 & NA (34), LOC100148890 \\
\hline D1 & A_44_P358492 & 1 & 1 & 0 & 0 & 0 & 0 & 0.18 and 0.23 & Farnesyl diphosphate farnesyl transferase 1 (Fdft1) \\
\hline D1 & A_42_P719350 & 1 & 1 & 0 & 0 & 0 & 0 & 0.18 and 0.23 & Glutamine rich 2 (Qrich2) (RGD1562974) \\
\hline D1 & A_44_P188275 & 1 & 1 & 0 & 0 & 0 & 0 & 0.19 and 0.20 & Sperm associated antigen 4 (Spag4) \\
\hline D1 & A_44_P368153 & 1 & 1 & 0 & 0 & 0 & 0 & 0.19 and 0.24 & Transglutaminase 3, E polypeptide (Tgm3) \\
\hline D1 & A_44_P1033758 & 1 & 1 & 0 & 0 & 0 & 0 & 0.20 and 0.22 & WD repeat domain 36 (Wdr36) \\
\hline D1 & A_44_P125681 & 1 & 1 & 0 & 0 & 0 & 0 & 0.20 and 0.23 & DNA (cytosine-5-)-methyltransferase 1 (Dnmt1) \\
\hline D1 & A_42_P621628 & 1 & 1 & 0 & 0 & 0 & 0 & 0.20 and 0.23 & Family with sequence similarity 63, member A (Fam63a) \\
\hline D1 & A_44_P135657 & 1 & 1 & 0 & 0 & 0 & 0 & 0.20 and 0.23 & Coiled-coil domain containing 113 (Ccdc113) \\
\hline D1 & A_44_P456599 & 1 & 1 & 0 & 0 & 0 & 0 & 0.20 and 0.24 & NA (40), TVAG_417060 \\
\hline D1 & A_44_P929214 & 1 & 1 & 0 & 0 & 0 & 0 & 0.22 and 0.23 & Notch1-induced protein (LOC493574) \\
\hline D1 & A_43_P12828 & 1 & 1 & 0 & 0 & 0 & 0 & 0.22 and 0.23 & Tektin 1 (Tekt1) \\
\hline D1 & A_42_P587156 & 1 & 1 & 0 & 0 & 0 & 0 & 0.22 and 0.23 & NIMA (never in mitosis gene a)-related expressed kinase 1 (Nek1) \\
\hline D1 & A_44_P260348 & 1 & 1 & 0 & 0 & 0 & 0 & 0.22 and 0.24 & NFA, hypothetical protein (LOC679115) \\
\hline D1 & A_43_P11723 & 1 & 1 & 0 & 0 & 0 & 0 & 0.22 and 0.25 & Guanylate cyclase activator 2a (guanylin) (Guca2a) \\
\hline D1 & A_44_P536832 & 1 & 1 & 0 & 0 & 0 & 0 & 0.23 and 0.23 & DENN/MADD domain containing 2A (Dennd2a) \\
\hline D1 & A_44_P829047 & 1 & 1 & 0 & 0 & 0 & 0 & 0.23 and 0.25 & Glucocorticoid modulatory element binding protein 1 (Gmeb1) \\
\hline D1 & A_44_P534844 & 1 & 1 & 0 & 0 & 0 & 0 & 0.24 and 0.25 & NA (38), LOC100040084 \\
\hline D1 & A_44_P524922 & 1 & 0 & 1 & 1 & 1 & 1 & $0.10-0.23$ & Malic enzyme 1, NADP(+)-dependent, cytosolic (Me1) \\
\hline D1 & A_44_P395421 & 1 & 0 & 1 & 0 & 1 & 0 & $0.13-0.21$ & NA (40), GL27102 \\
\hline D1 & A_44_P668980 & 1 & 0 & 1 & 0 & 0 & 0 & 0.01 and 0.15 & Gene model 1614 (Gm1614) \\
\hline D1 & A_44_P388673 & 2 & 0 & 2 & 0 & 0 & 0 & 0.02 and 0.20 & Haptoglobin (Hp) \\
\hline D1 & A_44_P464534 & 1 & 0 & 1 & 0 & 0 & 0 & 0.08 and 0.10 & *4, Insulin-like growth factor 1 receptor (Igf1r) \\
\hline D1 & A_42_P602822 & 1 & 0 & 1 & 0 & 0 & 0 & 0.08 and 0.22 & Ret proto-oncogene (Ret) \\
\hline D1 & A_44_P529581 & 1 & 0 & 1 & 0 & 0 & 0 & 0.09 and 0.23 & Oxidative stress induced growth inhibitor 1 (Osgin1) \\
\hline D1 & A_44_P510826 & 1 & 0 & 1 & 0 & 0 & 0 & 0.12 and 0.25 & Syntaxin $11($ Stx 11) \\
\hline D1 & A_44_P920649 & 1 & 0 & 1 & 0 & 0 & 0 & 0.13 and 0.20 & NA (38), RALGPS2 \\
\hline D1 & A_44_P1037456 & 1 & 0 & 1 & 0 & 0 & 0 & 0.14 and 0.21 & Procollagen, type XXII, alpha 1 (Col22a1) \\
\hline D1 & A_43_P15051 & 1 & 0 & 1 & 0 & 0 & 0 & 0.14 and 0.24 & NA (40), Tag-115 \\
\hline D1 & A_44_P794334 & 1 & 0 & 1 & 0 & 0 & 0 & 0.15 and 0.23 & NA (36), An07g02430 \\
\hline D1 & A_44_P105034 & 1 & 0 & 1 & 0 & 0 & 0 & 0.15 and 0.24 & Arachidonate 5-lipoxygenase (Alox5) \\
\hline D1 & A_44_P328796 & 1 & 0 & 1 & 0 & 0 & 0 & 0.16 and 0.17 & Sarcalumenin (Srl) \\
\hline D1 & A_44_P212575 & 1 & 0 & 1 & 0 & 0 & 0 & 0.16 and 0.21 & Butyrophilin-like 7 (Btnl7) \\
\hline D1 & A_42_P693821 & 1 & 0 & 1 & 0 & 0 & 0 & 0.19 and 0.23 & Fos-like antigen 1 (Fosl1) \\
\hline D1 & A_44_P592941 & 1 & 0 & 1 & 0 & 0 & 0 & 0.21 and 0.22 & NA (38), GG12284 \\
\hline D1 & A_44_P273291 & 1 & 0 & 0 & 2 & 0 & 1 & $0.07-0.23$ & Zinc finger protein 148 (Zfp148) \\
\hline D1 & A_44_P299167 & 1 & 0 & 0 & 0 & 1 & 0 & 0.13 and 0.18 & UDP-glucuronate decarboxylase 1 (Uxs1) \\
\hline D2 & A_44_P849944 & 0 & 2 & 2 & 1 & 1 & 1 & $0.15-0.21$ & Protease, serine, 35 (Prss35) \\
\hline D2 & A_44_P793901 & 0 & 1 & 1 & 0 & 0 & 1 & $0.14-0.17$ & NA (36), GSPATT00022597001 \\
\hline D2 & A_43_P21610 & 0 & 1 & 1 & 0 & 0 & 0 & 0.03 and 0.03 & Serine (or cysteine) peptidase inhibitor, clade C 1 (Serpinc1) \\
\hline D2 & A_44_P541548 & 0 & 1 & 1 & 0 & 0 & 0 & 0.11 and 0.16 & *5, Angiotensin II receptor- associated protein (Agtrap) \\
\hline D2 & A_44_P185703 & 0 & 1 & 1 & 0 & 0 & 0 & 0.13 and 0.13 & Iron-sulfur cluster assembly 1 homolog (Isca1) \\
\hline D2 & A_43_P15517 & 0 & 1 & 1 & 0 & 0 & 0 & 0.15 and 0.19 & Jun D proto-oncogene (Jund) \\
\hline D2 & A_44_P716655 & 0 & 1 & 1 & 0 & 0 & 0 & 0.17 and 0.19 & NFA, RIKEN cDNA 1700012B09 (RGD1561795) \\
\hline $\mathrm{D} 2$ & A_44_P337441 & 0 & 1 & 1 & 0 & 0 & 0 & 0.23 and 0.23 & Lipase, endothelial (Lipg) \\
\hline D2 & A_44_P813198 & 0 & 1 & 0 & 1 & 1 & 0 & $0.11-0.23$ & NA (38), GD19242 \\
\hline D3 & A_44_P306008 & 0 & 0 & 1 & 1 & 1 & 0 & $0.07-0.25$ & EP300 interacting inhibitor of differentiation 1 (Eid1) \\
\hline D4 & A_44_P279419 & 0 & 0 & 0 & 1 & 1 & 1 & $0.10-0.25$ & Calcium channel, voltage-dependent, a2/d1 (Cacna2d1) \\
\hline D4 & A_44_P503215 & 0 & 0 & 0 & 1 & 1 & 1 & $0.11-0.12$ & CD36 (RGD1562323), fatty acid translocase \\
\hline D4 & A_44_P348527 & 0 & 0 & 0 & 1 & 1 & 1 & $0.12-0.23$ & Pumilio 1 (Pum1) \\
\hline D4 & A_44_P827889 & 0 & 0 & 0 & 1 & 1 & 1 & $0.14-0.23$ & misc_RNA(CNOT6L) \\
\hline D4 & A_44_P153063 & 0 & 0 & 0 & 1 & 1 & 1 & $0.17-0.21$ & Peptidylglycine $\alpha$-amidating monooxygenase (Pam) \\
\hline D4 & A_44_P202450 & 0 & 0 & 0 & 1 & 1 & 1 & $0.18-0.23$ & NFA, C50H11.1 (LOC498962) \\
\hline D4 & A_44_P147751 & 0 & 0 & 0 & 1 & 1 & 1 & $0.22-0.24$ & Ring finger protein 4 (Rnf4) \\
\hline
\end{tabular}


Table IV. Continued.

\begin{tabular}{llllllllll}
\hline Gr & Probe ID & $6 \mathrm{H}$ & $6 \mathrm{~S}$ & $6 \mathrm{M}$ & $9 \mathrm{H}$ & $9 \mathrm{~S}$ & $9 \mathrm{M}$ & Fold-change & Gene or protein name (symbol) \\
\hline D4 & A_44_P440082 & 0 & 0 & 0 & 1 & 1 & 0 & 0.14 and 0.15 & Stearoyl-CoA desaturase (delta-9-desaturase) (Scd) \\
D4 & A_44_P360664 & 0 & 0 & 0 & 1 & 1 & 0 & 0.17 and 0.17 & Prolactin receptor (Prlr) \\
D4 & A_44_P165151 & 0 & 0 & 0 & 1 & 1 & 0 & 0.20 and 0.23 & Platelet-activating factor acetylhydrolase, 1b, 1 (Pafah1b1) \\
D4 & A_44_P397657 & 0 & 0 & 0 & 1 & 1 & 0 & 0.20 and 0.23 & Apolipoprotein C-IV (Apoc4) \\
D4 & A_44_P242773 & 0 & 0 & 0 & 1 & 1 & 0 & 0.21 and 0.21 & RAB10, member RAS oncogene family (Rab10) \\
D4 & A_44_P242664 & 0 & 0 & 0 & 1 & 1 & 0 & 0.21 and 0.22 & Proteasome subunit, $\beta$ type 3 (Psmb3) \\
D4 & A_44_P233399 & 0 & 0 & 0 & 1 & 1 & 0 & 0.21 and 0.22 & Niemann-Pick disease type C2 (Npc2) \\
D4 & A_44_P298709 & 0 & 0 & 0 & 1 & 1 & 0 & 0.22 and 0.22 & Coagulation factor II receptor (F2r) \\
D4 & A_44_P364929 & 0 & 0 & 0 & 1 & 1 & 0 & 0.22 and 0.22 & ADP-ribosylation factor-like 6 interacting protein 1 (Ar16ip1) \\
D4 & A_44_P329712 & 0 & 0 & 0 & 1 & 1 & 0 & 0.22 and 0.23 & NA (40), BBOV_IV009770 \\
D4 & A_44_P340805 & 0 & 0 & 0 & 1 & 0 & 1 & 0.12 and 0.16 & NA (40), 2010011I20Rik \\
D5 & A_44_P326584 & 0 & 0 & 0 & 0 & 1 & 1 & 0.15 and 0.22 & Complement component 7 (C7) \\
D5 & A_44_P353041 & 0 & 0 & 0 & 0 & 1 & 1 & 0.21 and 0.24 & NA (36), OSTLU_52010 \\
\hline
\end{tabular}

Gr, down-regulated D1, D2, D3, D4 and D5 groups; Numbers 0, 1, 2 and 3 indicate the number of probes showing positive results. Fold-change, <1/4-fold down-regulation in at least two different experiments. Other symbols or abbreviations are as described in Tables II and III.

\section{Discussion}

General considerations. Analysis of the relationship between the gene expression profiles of the three SHR substrains at 6 and 9 weeks of age yielded strong evidence of a heritable influence on gene expression in these substrains (Table I). The number of probes corresponding to up-regulated genes at 6 weeks of age clearly increased from SHR via SHRSP to M-SHRSP; this order reflects the genesis of the three SHR substrains (Table I) $(3,4,19)$. By contrast, the number of probes corresponding to the down-regulated genes in SHR at 6 weeks of age was as high as 4,407, and was markedly decreased to 149 in SHRSP and to 74 in M-SHRSP (Table I). These results suggest that the mutations introduced into SHRSP and M-SHRSP effectively inhibited the down-regulation of genes in these SHR, and promoted hypertension in SHRSP and M-SHRSP. Some of the genes down-regulated in SHR, but not in SHRSP and M-SHRSP, at 6 weeks of age may play roles in alleviating the stroke-prone and/or malignant symptoms observed in SHRSP and M-SHRSP. An analysis of the genes related to these symptoms will be undertaken in our future studies.

Candidate genes involved in the genesis of hypertension among the up-regulated genes. The $\mathrm{U} 1$ and $\mathrm{U} 2$ groups are expected to include the most probable candidate genes involved in hypertension pathogenesis. They consist of 20 annotated and 8 non-annotated genes. Among the annotated genes, at least 4 candidate genes were identified (Table III, *1 to *4): (i) Spock2 was not only up-regulated in all SHR substrains at 6 and 9 weeks of age, but is also reported to be an effector of angiotensin II signaling, which plays a pivotal role in BP control. It is thus one of the most probable candidate genes involved in the pathogenesis of hypertension (10). (ii) Kynu is reported to be among the candidate genes that contribute to hypertension in SHR, since the injection of kynurenic acid into the rostral ventrolateral medulla of SHR has been shown to decrease arterial BP (11). Increased expression of Kynu is thought to decrease kynurenine levels and increase arterial BP. (iii) Rgs2 is up-regulated in the adrenal glands of SHRSP and M-SHRSP at 6 weeks of age (Table III), and has been reported to mediate vascular smooth muscle relaxation and BP (12). Notably, Rgs $2^{-/-}$mice develop marked hypertension, and their blood vessels show enhanced contraction (12). (iv) Gjal, called connexin43 (Cx43) in humans, is involved in the control of cell-to-cell communication and is thought to modulate the contractility of the vascular wall and the electrical coupling of cardiomyocytes (13).

Other than these 4 genes, several annotated genes, such as nuclear receptor subfamily 4 (Nr4a3), FBJ osteosarcoma oncogene homolog (c-Fos) and jun B proto-oncogene (Junb) (Table III, U2 and U3) are known to be up-regulated by angiotensin II and affect BP through signal transduction and cellular growth (20). We presume that the candidate genes are not included in the U3, U4 and U5 groups. However, these groups do apparently include genes related to BP control, such as cysteine-rich angiogenic inducer 61 (Cyr61), purinergic receptor P2Y G-protein coupled 2 (P2ry2), Junb, C-type natriuretic peptide precursor $(\mathrm{Cnp})$ and transcriptional regulating factor 1 (Trerf1) (21-25). Our interpretation is that though these genes are not the primary candidate genes, their expression is affected by the candidate genes, thus contributing to deteriorating hypertension.

Candidate genes involved in the genesis of hypertension among the down-regulated genes. The D1 and D2 groups are expected to include the most probable candidate genes involved in hypertension pathogenesis. They consist of 77 annotated and 30 non-annotated genes (Table IV). Among the annotated genes, at least 5 candidate genes were identified (Table IV, ${ }^{*} 1$ to ${ }^{*} 5$ ): (i) Uts 2 encodes a peptide ligand that acts on the $\mathrm{G}$ protein-coupled urotensin receptor and elicits long-term vasoconstriction (14). Human urotensin II promotes hypertension and atherosclerotic cardiovascular diseases (26). Plasma urotensin II levels are elevated in patients with vascular endothelial dysfunction-related diseases such as 
essential hypertension, diabetes mellitus, atherosclerosis, ischemic heart disease and heart failure (26). (ii) Ephx2 leads males homozygous for a targeted null mutation to display a significant reduction in BP, both in the presence and absence of dietary salt loading (15). Monti et al (27) identified Ephx2 as a heart failure susceptibility gene in SHHF rats derived from SHR Koletsky rats. (iii) Apln and its receptor share similarities in structure and anatomical distribution with angiotensin II and the angiotensin AT1 receptor, providing clues regarding the physiological functions of this novel signaltransduction system (16). It has now been established that the Apln system plays a role in lowering BP, as a potent cardiac inotrope, in modulating pituitary hormone release and food and water intake, in stress activation, and as a novel adipokine that is excreted from fat cells and regulates insulin. Given its broad array of physiological roles, Apln has attracted much interest as a target for novel therapeutic research and drug design (15) (Table IV, D1). (iv) Igflr is known to cause direct vasodilation via a nitric oxide-dependent pathway $(17,28)$. (v) Agtrap encodes a transmembrane protein and is a modulator of angiotensin II signaling (18). Overexpression of the novel Agtrap induces cellular hypertrophy in cultured rat vascular smooth muscle and renal proximal tubular cells.

Other than these 5 genes, several genes listed in the D1 and D2 groups (Table IV) are known to affect BP. For example, haptoglobin $(H p)$ is associated with increased $\mathrm{BP}$ (29), arachidonate 5-lipoxygenase (Alox5) plays a critical role in the progression of pulmonary hypertension in rats (30), and endothelial lipase ( Lipg) probably has a role in the pathophysiology of vascular diseases (31). The Niemann-Pick disease type C2 (Npc2), coagulation factor II receptor $(F 2 r)$ and complement component $7(C 7)$ genes reportedly participate in BP control (32-34). However, since they are classified into the D4 and D5 groups, we presume that they are not the primary candidate genes.

Also of note, in the $\mathrm{D} 1$ group, the $\mathrm{DEAD} / \mathrm{H}$ box polypeptide $11(D d x 11)$ and ER-Golgi intermediate compartment protein 1 (Ergicl) genes $(35,36)$ showed down-regulation among all SHR substrains at 6 and 9 weeks of age (Table IV, D1). These genes are not currently directly associated with hypertension. However, their expression profiles suggest their participation in the genesis of hypertension in the SHR substrains.

Other possible candidate genes for hypertension in SHRs. Although more detailed analyses are necessary, it is anticipated that further investigation of the non-annotated genes will provide insights into their specific roles in the etiology of hypertension. The U1 and U2 groups included 8 functionally non-annotated genes, and the D1 and D2 groups 30 functionally non-annotated genes. Their expression profiles suggest that they encode unknown proteins with functions related to BP control.

Although current gene expression arrays permit the simultaneous analysis of thousands of rat genes, this method is not yet capable of addressing all functional genes within the genome. However, as rat genome annotation progresses and arrays continue to improve in their extent of genomic coverage, a more complete analysis should become possible. Our current approach identified at least 9 genes, including Куnu (10) and Ephx2 (26), as the most probable candidate genes causing hypertension in SHR substrains. Kynu and Ephx2 have previ- ously been demonstrated to be responsible for hypertension in SHR; however, we believe that the remaining genes also participate in the genesis of hypertension in SHR substrains.

\section{Acknowledgements}

This work was supported by a Japanese Grant-in-Aid for Scientific Research (no. 19500699), a MEXT grant to private universities to cover current expenses, a fund from the MishimaKaiun Memorial Foundation, a donation from Ueshima Coffee Co., Ltd., and by the Kinki University School of Medicine. We thank Dr Etsuro Yamanishi, President Emeritus of Hirakata Ryoikuen, for his constant support and encouragement, and the National Center for Biotechnology Information, USA and the DNA Data Bank of Japan for access to their network servers.

\section{References}

1. McBride MW, Charchar FJ, Graham D, et al: Functional genomics in rodent models of hypertension. J Physiol 554: 56-63, 2004.

2. Okamoto K and Aoki K: Development of a strain of spontaneously hypertensive rat. Jpn Circ J 27: 282-293, 1963.

3. Okamoto K, Yamori $\mathrm{Y}$ and Nagaoka A: Establishment of the stroke-prone spontaneously hypertensive rats (SHR). Circ Res 34 (Suppl 1): 143-153, 1974.

4. Okamoto K, Yamamoto K, Morita N and Ohta Y: Establishment and characteristics of rat with precocious and severe hypertension (M-SHRSP). Acta Med Kinki Univ 10: 73-95, 1985.

5. Friese RS, Mahboubi P, Mahapatra NR, et al: Common genetic mechanisms of blood pressure elevation in two independent rodent models of human essential hypertension. Am J Hypertens 18: 633-652, 2005.

6. Simon R, Lam A, Li M-C, et al: Analysis of gene expression data using BRB-array tools. Cancer Informatics 3: 11-17, 2007.

7. Altschul SF, Thomas LM, Alejandro AS, et al: Gapped BLAST and PSI-BLAST: a new generation of protein database search programs. Nucleic Acids Res 25: 3389-3402, 1977.

8. Tanase H, Suzuki Y, Ooshima A, et al: Genetic analysis of blood pressure in spontaneously hypertensive rats. Jpn Circ J 34: 1197-1212, 1970.

9. Yen TT, Yu PL, Roeder H and Willard PW: A genetic study of hypertension in Okamoto-Aoki spontaneously hypertensive rats. Heredity 33: 309-316, 1974.

10. Socha MJ, Manhiani M, Said N, et al: Secreted protein acidic and rich in cysteine deficiency ameliorates renal inflammation and fibrosis in angiotensin hypertension. Am J Pathol 171: 1104-1112, 2007.

11. Mizutani K, Sugimoto K, Okuda T, et al: Kynureninase is a novel candidate gene for hypertension in spontaneously hypertensive rats. Hypertens Res 25: 135-140, 2002.

12. Tang M, Wang G, Lu P, et al: Regulator of G-protein signaling-2 mediates vascular smooth muscle relaxation and blood pressure. Nat Med 9: 1506-1512, 2003.

13. Haefliger JA, Meda P, Formenton A, et al: Aortic connexin43 is decreased during hypertension induced by inhibition of nitric oxide synthase. Arterioscler Thromb Vasc Biol 19: 1615-1622, 1999.

14. Tsai CS, Loh SH, Liu JC, et al: Urotensin II-induced endothelin-1 expression and cell proliferation via epidermal growth factor receptor transactivation in rat aortic smooth muscle cells. Atherosclerosis 206: 86-94, 2009.

15. Choi MY, Romer AI, Hu M, et al: A dynamic expression survey identifies transcription factors relevant in mouse digestive tract development. Development 133: 4119-4129, 2006.

16. Lee DK, George SR and O'Dowd BF: Unravelling the role of the apelin system: prospective therapeutic applications in heart failure and obesity. Trend Pharmacol Sci 27: 190-194, 2006.

17. Cruzado MC, Risler NR, Miatello RM, et al: Vascular smooth muscle cell $\mathrm{NAD}(\mathrm{P}) \mathrm{H}$ oxidase activity during the development of hypertension: effect of angiotensin II and role of insulin-like growth factor-1 receptor transactivation. Am J Hypertens 18: 81-87, 2005.

18. Lopez-Ilasaca M, Liu X, Tamura K and Dzau VJ: The angiotensin II type I receptor-associated protein, ATRAP, is a transmembrane protein and a modulator of angiotensin II signaling. Mol Biol Cell 14: 5038-5050, 2003. 
19. Okamoto K, Yamamoto N, Morita Y, et al: Establishment and use of the M strain of stroke-prone spontaneously hypertensive rat. J Hypertens (Suppl 4): 21-24, 1986.

20. Nogueira EF, Vargas CA, Otis M, et al: Angiotensin-II acute regulation of rapid response genes in human, bovine and rat adrenocortical cells. J Mol Endocrinol 39: 365-374, 2007.

21. Grzeszkiewicz TM, Lindner V, Chen N, et al: The angiogenic factor cysteine-rich 61 (CYR61, CCN1) supports vascular smooth muscle cell adhesion and stimulates chemotaxis through integrin alpha(6)beta(1) and cell surface heparan sulfate proteoglycans. Endocrinology 143: 1441-1450, 2002.

22. Wang Z, Nakayama T, Sato N, et al: The purinergic receptor P2Y, G-protein coupled, 2 (P2RY2) gene associated with essential hypertension in Japanese men. J Hum Hypertens: Aug 27, 2009 (E-pub ahead of print)

23. Rose P, Bond J, Tighe S, et al: Genes overexpressed in cerebral arteries following salt-induced hypertensive disease are regulated by angiotensin II, JunB and CREB. Am J Physiol Heart Circ Physiol 294: H1075-H1085, 2008.

24. Passino C, Del Ry S, Severino S, et al: C-type natriuretic peptide expression in patients with chronic heart failure: effects of aerobic training. Eur J Cardiovasc Prev Rehabil 15: 168-172, 2008

25. Gizard F, Robillard R, Barbier O, et al: TReP-132 controls cell proliferation by regulating the expression of the cyclin-dependent kinase inhibitors p21WAF1/Cip1 and p27Kip1. Mol Cell Biol 25: 4335-4348, 2005

26. Watanabe T, Arita S, Shiraishi Y, et al: Human urotensin II promotes hypertension and atherosclerotic cardiovascular diseases. Curr Med Chem 16: 550-563, 2009.

27. Monti J, Fischer J, Paskas S, et al: Soluble epoxide hydrolase is a susceptibility factor for heart failure in a rat model of human disease. Nat Genet 40: 529-537, 2008.
28. Pete G, Hu Y, Walsh M, et al: Insulin-like growth factor-I decreases mean blood pressure and selectively increases regional blood flow in normal rats. Proc Soc Exp Biol Med 213: 187-192, 1996.

29. Havlik RJ: Predictors of hypertension. Population studies. Am J Hypertens 4: S586-S589, 1991.

30. Osher E, Weisinger G, Limor R, et al: The 5 lipoxygenase system in the vasculature: emerging role in health and disease. Mol Cell Endocrinol 252: 201-206, 2006.

31. Williams RR, Hunt SC, Hasstedt SJ, et al: Multigenic human hypertension: evidence for subtypes and hope for haplotypes. J Hypertens (Suppl 8): 39-46, 1990.

32. Garver WS and Heidenreich RA: The Niemann-Pick C proteins and trafficking of cholesterol through the late endosomal/lysosomal system. Curr Mol Med 2: 485-505, 2002.

33. Hermann GE, van Meter MJ, Rood JC, et al: Proteinaseactivated receptors in the nucleus of the solitary tract: evidence for glial-neural interactions in autonomic control of the stomach. J Neurosci 29: 9292-9300, 2009.

34. Nauta AJ, Daha MR, Tijsma O, et al: The membrane attack complex of complement induces caspase activation and apoptosis. Eur J Immunol 32: 783-792, 2002.

35. Vasa-Nicotera M, Brouilette S, Mangino M, et al: Mapping of a major locus that determines telomere length in humans. Am J Hum Genet 76: 147-151, 2005.

36. Breuza L, Halbeisen R, Jen P, et al: Proteomics of endoplasmic reticulum-Golgi intermediate compartment (ERGIC) membranes from brefeldin A-treated HepG2 cells identifies ERGIC-32, a new cycling protein that interacts with human Erv46. J Biol Chem 279: 47242-47253, 2004. 